\title{
Relationship Between FDG Uptake and Neutrophil/Lymphocyte Ratio in Patients with Invasive Ductal Breast Cancer
}

\author{
TAKAAKI FUJII, KEIKO YANAI, SHOKO TOKUDA, YUKO NAKAZAWA, SASAGU KUROZUMI, \\ SAYAKA OBAYASHI, REINA YAJIMA, TOMOKO HIRAKATA and KEN SHIRABE \\ Division of Breast and Endocrine Surgery, Department of General Surgical Science, \\ Graduate School of Medicine, Gunma University, Gunma, Japan
}

\begin{abstract}
Background: ${ }^{18}$ F-Fluorodeoxyglucose-positronemission tomography (FDG-PET) is used to evaluate the glucose metabolic rates of tumors. Several studies have reported that high FDG uptake is predictive of poor prognosis and aggressive features in patients with breast cancer. FDG uptake is influenced by many factors, including inflammation. In this study, the relationship between FDG uptake and neutrophil/lymphocyte ratio $(N L R)$, which is an indicator of systemic inflammation, was investigated. Patients and Methods: A retrospective investigation of the cases of 143 consecutive patients with invasive ductal carcinoma who had undergone surgery and FDG-PET preoperatively. PET was evaluated using standardized uptake value $\max \left(S U V_{\text {max }}\right)$. The median $S U V_{\max }$ was 2.5 (range=0-10.5). The cases were divided into two groups based on the value of $S U V_{\max }$ : low $(<2.5)$ and high $(\geq 2.5)$. The relationships between $S U V_{\max }$ and clinicopathological features, including NLR, were investigated. Results: Among the 143 patients, 73 (51.0\%) had high $S U V_{\max }$ in the primary tumor. The analysis revealed that large tumor size $(p<0.001)$, high nuclear grade $(p<0.001)$, the presence of lymphovascular invasion $(p<0.001)$, high $C$-reactive protein $(p=0.046)$ and high NLR $(p<0.001)$ were significantly associated with high $S U V_{\max }$ in the primary tumor. $S U V_{\max }$ and $N L R$ were significantly positively correlated $(r=0.323, p<0.001)$. Among the 70 cases with low $S U V_{\text {max }}$, there was no recurrent disease, while out of the 73 cases with high $S U V_{\text {max }}$ had disease recurrence. It is interesting to note that
\end{abstract}

Correspondence to: Takaaki Fujii, MD, Ph.D., FACS, Department of General Surgical Science, Graduate School of Medicine, Gunma University, 3-39-22 Showa-machi, Maebashi, Gunma 371-8511, Japan. Tel: +81 0272208224, Fax: +81 0272208230, e-mail: ftakaaki@gunma-u.ac.jp

Key Words: FDG-PET, FDG uptake, breast cancer, neutrophil/ lymphocyte ratio, inflammation. the group with high $S U V_{\max }$ and low NLR had no recurrent disease. Conclusion: The present study demonstrated that the finding of high preoperative FDG uptake in breast cancer may be reflective of poor prognosis and that a high NLR may be predictive of aggressive features among patients with breast cancer. On the other hand, among patients with breast cancer with high $S U V_{\max }$ in the primary tumor, it will be useful to identify those with a low $N L R$ in order to improve prognostic accuracy.

There is increasing evidence to show that the presence of a systemic inflammatory response is associated with poor survival in multiple types of cancers (1-7). Cancer progression and prognosis are affected by the patient's inflammatory response in the tumor microenvironment $(1,2)$. Recently, the neutrophil/lymphocyte ratio (NLR) was found to be a prognostic factor in breast cancer. It is of interest that the presence of a systemic inflammatory response, as evidenced by an elevated NLR, has been shown to predict recurrence and overall survival in patients with breast cancer (2, 8-12).

In recent years, the clinical applications of positronemission tomography (PET) have grown explosively. PET using ${ }^{18} \mathrm{~F}$-fluorodeoxyglucose (FDG) is a non-invasive wholebody imaging technique used to evaluate different kinds of malignancies, including breast cancer, for tumor staging and restaging, detecting recurrence, and monitoring treatment responses (13-21). FDG-PET measures glucose metabolism, which reflects the biological aggressiveness of tumors (21-26). Several studies have reported that high FDG uptake is predictive of poor prognosis and aggressive features in patients with breast cancer (21-26). FDG uptake is influenced by many factors, including inflammation, yet no published study, to our knowledge, has assessed the association between FDG uptake and NLR in breast cancer cases, even though both these measures represent inflammation.

In this study, we investigated the relationship between FDG uptake and the NLR at baseline in patients with breast cancer. 


\section{Patients and Methods}

Patients. We retrospectively investigated the cases of 143 consecutive patients with primary breast cancer who had undergone FDG-PET preoperatively at Gunma University between January 2010 and October 2015. All patients subsequently underwent radical breast surgery. Patients with synchronous bilateral breast cancer or clinical signs of infection or other inflammatory conditions preoperatively, including pneumonia or articular rheumatism, were excluded from the study. Patients with incomplete clinical information and male patients were excluded. Patients underwent FDG-PET/computed tomography (CT) as part of the routine standard of care without deviating from the main protocol. The maximum standardized FDG uptake value $\left(\mathrm{SUV}_{\max }\right)$ of primary tumors was calculated in a routine clinical fashion. Written consent was obtained from all patients for the use of their records and imaging in future studies.

The details extracted from the database were age, histological type, size of invasive primary tumor, size of ductal spread, lymphatic or vascular invasion, nuclear grade, estrogen receptor (ER) and progesterone receptor (PgR) expression status, human epidermal growth factor receptor 2 (HER2) score of the primary tumor, axillary lymph node status, serum C-reactive protein (CRP), serum carcinoembryonic antigen (CEA), hemogram parameters (neutrophils and lymphocytes), and $\mathrm{SUV}_{\max }$ of the primary tumor and visibility of detected lesion by FDG-PET. The ER and PgR statuses were assessed by ALLRED scores, with an ALLRED score of 3 or higher indicating ER and PgR positivity (27). NLR was defined as the absolute neutrophil count divided by the absolute lymphocyte count. The relationships between $\mathrm{SUV}_{\max }$ and clinicopathological features, including NLR, were investigated. The median $\mathrm{SUV}_{\max }$ was 2.5 (range=0-10.5). Therefore, the cases were divided into two groups based on $\mathrm{SUV}_{\max }$ value: low $(<2.5)$ and high $(\geq 2.5)$.

Statistical analysis. The breast cancer cases were divided into two groups on the basis of FDG uptake in the primary tumor. A univariate statistical analysis was carried out using Fisher's exact test or the chi-squared test with Yates' correction. To compare the two groups, Student's $t$-test was used. Differences were considered significant when $p<0.05$. Relapse-free survival (RFS) was calculated using the Kaplan-Meier method. The log-rank test was used to evaluate differences in overall survival and the recurrencefree interval. Differences were considered significant when $p<0.05$.

\section{Results}

In total, 143 cases were included in the analysis. The median $\mathrm{SUV}_{\text {max }}$ was 2.5 (range=0-10.5). Among the143 patients, 73 $(51.0 \%)$ had high $\mathrm{SUV}_{\max }$ in the primary tumor. The 143 cases with breast cancer were divided into two groups based on $\mathrm{SUV}_{\text {max }}$ in the primary tumor. Table I shows the patient characteristics and summarizes the results of the univariate analysis conducted to determine the relationships between $\mathrm{SUV}_{\text {max }}$ in the primary tumor and clinicopathological variables.

The univariate analysis revealed that large tumor size $(p<0.001)$, high nuclear grade $(p<0.001)$, the presence of lymphovascular invasion $(p<0.001)$, high CRP $(p=0.046)$ and high NLR $(p<0.001)$ were significantly associated with high $\mathrm{SUV}_{\text {max }}$ in the primary tumor. SUV $\mathrm{max}_{\text {max }}$ and NLR were positively correlated $(\mathrm{r}=0.323, p<0.001)$.

The RFS shown by the Kaplan-Meier curves was significantly shorter for patients with high $\operatorname{SUV}_{\text {max }}(p=0.013)$ (Figure 1). Among the 70 cases with low $\mathrm{SUV}_{\max }$, there was no recurrent disease, while six out of the 73 cases with high $\mathrm{SUV}_{\text {max }}$ had disease recurrence. The overall median followup period was 48.9 months (range=9.6-94.7 months).

It is interesting to note that the group with high $\mathrm{SUV}_{\text {max }}$ and low NLR $($ NLR $<1.8)$ had no recurrent disease, although there were no differences in any clinicopathological features between the group with high NLR and high $\mathrm{SUV}_{\text {max }}$ and that with low NLR with high $\mathrm{SUV}_{\max }$ (Table II).

\section{Discussion}

Inflammation in patients with cancer is a significant problem due to a variety of mechanisms involving the tumor and the host response to the tumor. Several studies have reported that high FDG uptake is predictive of poor prognosis and aggressive features in patients with breast cancer (21-26). FDG-PET provides biological information about a tumor's growth potential and shows inflammation. Recently, NLR was reported to be a prognostic factor in breast cancer. NLR is considered as important as CRP in the inflammatory assessment. It is of interest that some authors have reported that NLR reflects systemic inflammation and prognosis in patients with cancer (2, 8-12). The key observations of the present study can be summarized as follows: i) among various clinicopathological characteristics, high $\mathrm{SUV}_{\max }$ was associated with high NLR; ii) high $\mathrm{SUV}_{\max }$ was associated with disease recurrence in patients with operable breast cancer; iii) the group with high $\mathrm{SUV}_{\max }$ and low NLR had no recurrent disease. Our results suggest that FDG uptake may be predictive of inflammation in addition to aggressive features among patients with breast cancer. These findings provide evidence that high $\mathrm{SUV}_{\max }$ is a useful predictor of disease recurrence in patients with operable breast cancer, but cases with low NLR despite high $\mathrm{SUV}_{\text {max }}$ in the primary tumor may have a lower risk of recurrent disease.

$\mathrm{SUV}_{\text {max }}$ is used as a semi-quantitative indicator of FDG uptake as it is influenced by many factors, including glucose transporter expression, viable cell number, tumor perfusion and inflammatory cells $(14,18,28)$. FDG-PET measures local glucose metabolism and may reflect local inflammation of cancer. For the evaluation of breast cancer, it is important to understand not only systemic inflammatory response but also local inflammatory response represented by FDG avidity. As components of systemic inflammatory response, lymphocytes and neutrophils are increasingly being recognized as having an important role in cancer progression. NLR is determined to indicate systemic inflammation. From our findings, high $\mathrm{SUV}_{\text {max }}$ with high NLR may reflect aggressive tumor features 
Table I. Patient characteristics and clinicopathological features associated with ${ }^{18} \mathrm{~F}$-fluorodeoxyglucose (FDG) uptake in primary breast cancer.

\begin{tabular}{lllr}
\hline & \multicolumn{2}{c}{ FDG uptake } \\
\cline { 2 - 3 } & Low (n=70) & High (n=73) & $p$-Value \\
\hline Age, mean \pm SD (years) & $60.3 \pm 11.3$ & $57.6 \pm 12.5$ & 0.906 \\
Tumor size (mm) & $14.4 \pm 9.1$ & $23.0 \pm 9.9$ & $<0.001$ \\
Nodal metastasis-positive (n) & 11 & 21 & 0.095 \\
ER-positive (n) & 63 & 58 & 0.064 \\
PgR-positive (n) & 53 & 55 & 0.886 \\
HER2-positive (n) & 8 & 17 & 0.049 \\
Nuclear grade 3 (n) & 14 & 42 & $<0.001$ \\
Lymphatic invasion-positive (n) & 17 & 41 & $<0.001$ \\
Vascular invasion-positive (n) & 5 & 23 & $<0.001$ \\
NLR, mean \pm SD & $2.08 \pm 0.91$ & $2.83 \pm 1.65$ & $<0.001$ \\
CEA (ng/mI), mean \pm SD & $2.26 \pm 1.67$ & $2.61 \pm 2.97$ & 0.191 \\
CRP (mg/mI), mean \pm SD & $0.05 \pm 0.06$ & $0.20 \pm 0.73$ & 0.046 \\
\hline
\end{tabular}

$\mathrm{n}$, Number of patients; ER, estrogen receptor; PgR, progesterone receptor; HER2, human epidermal growth factor receptor 2; NLR, neutrophil:lymphocyte ratio; CRP, C-reactive protein; CEA, carcinoembryonic antigen.

Table II. Patient characteristics and clinicopathological features associated with neutrophil:lymphocyte ratio $(N L R)$ in patients with high ${ }^{18}$ F-fluorodeoxyglucose (FDG) uptake.

\begin{tabular}{lccc}
\hline & \multicolumn{2}{c}{ NLR } \\
\cline { 2 - 3 } & Low (n=16) & High (n=57) & $p$-Value \\
\hline Age, mean \pm SD (years) & $57.6 \pm 11.6$ & $57.7 \pm 12.8$ & 0.519 \\
Tumor size (mm) & $22.4 \pm 8.8$ & $23.1 \pm 10.2$ & 0.600 \\
Nodal metastasis-positive (n) & 2 & 19 & 0.090 \\
ER-positive (n) & 15 & 43 & 0.100 \\
PgR-positive (n) & 14 & 41 & 0.173 \\
HER2-positive (n) & 2 & 15 & 0.210 \\
Nuclear grade 3 (n) & 12 & 30 & 0.093 \\
Lymphatic invasion-positive (n) & 6 & 35 & 0.079 \\
Vascular invasion-positive (n) & 5 & 18 & 0.617 \\
SUV & $5.04 \pm 2.06$ & $5.19 \pm 2.70$ & 0.587 \\
CEA (ng/ml), mean \pm SD & $3.06 \pm 3.41$ & $2.48 \pm 2.69$ & 0.273 \\
CRP (mg/ml), mean \pm SD & $0.06 \pm 0.10$ & $0.24 \pm 0.82$ & 0.934 \\
\hline
\end{tabular}

n, Number of patients; ER, estrogen receptor; PgR, progesterone receptor; HER2, human epidermal growth factor receptor 2; NLR, neutrophil:lymphocyte ratio; CRP, C-reactive protein; CEA, carcinoembryonic antigen.

and systemic inflammation, which strongly predict poor prognosis in patients with breast cancer. On the other hand, a high $\mathrm{SUV}_{\max }$ without a high NLR may predict local inflammation at the tumor, which may reflect immunoreaction to the tumor.

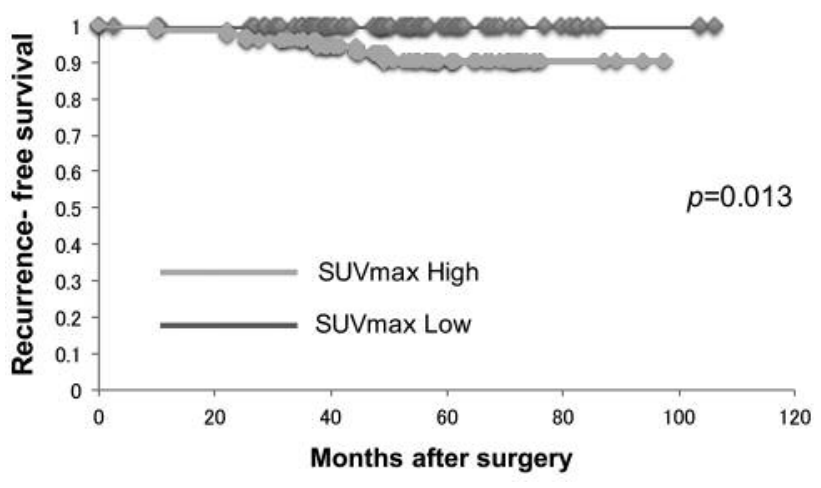

Figure 1. Recurrence-free survival curves according to standardized uptake value $\max \left(S U V_{\max }\right)$ of the primary tumor. The recurrence-free interval for the group with high $S U V_{\max }$ was significantly shorter than that with low $S U V_{\max }(p=0.013)$. The overall median follow-up period was 48.9 months (range=9.6-94.7 months).

An elevated NLR is associated with adverse survival probabilities in multiple cancer types, including breast cancer. However, evidence of the prognostic role of NLR in breast cancer is relatively controversial (12, 29). Inflammation is associated with many factors, and an elevated NLR is not only induced by the cancer environment. In our study, there was no recurrence in patients with low $\mathrm{SUV}_{\max }$ with high NLR. Therefore, an elevated NLR without FDG uptake in the primary tumor may not pose an increased risk of recurrent disease.

Cancer progression and prognosis are affected by many factors, including the host's inflammatory response or immunological response in the tumor microenvironment (1, 2). From our findings, $S U V_{\max }$ represents the local reaction of the tumor and NLR predicts the systemic inflammatory reaction, including the response to the tumor; it is considered that the combination of $\mathrm{SUV}_{\max }$ and NLR findings is effective for predicting local and systemic tumor microenvironments and predicting prognoses of patients with breast cancer.

This study had several potential limitations, the major ones being that it was a retrospective analysis and that the number of cases was relatively small. Additional research is needed to explore other benefits and drawbacks of NLR and FDG-PET evaluation of primary breast cancer. However, to the best of our knowledge, this is the first report to describe the relationship between FDG uptake and NLR in breast cancer. NLR as a predictor in patients with primary breast cancer with

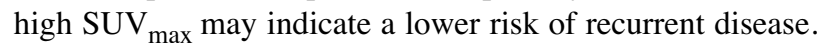

In conclusion, we demonstrated that the finding of a high preoperative $\mathrm{SUV}_{\max }$ in primary breast cancer is effective for predicting poor prognosis among patients. On the other hand, among those with high $\mathrm{SUV}_{\max }$ in the primary tumor, it will be useful to identify the subset of patients with low NLR to improve prognostic accuracy. Analyses from large 
randomized trials are warranted to evaluate the relationship between the combination of these two factors and disease recurrence.

\section{Competing Interest}

The Authors declare that they have no competing financial interests in regard to this study.

\section{Acknowledgements}

The Authors would like to thank Y. Saitoh, F. Takata and H. Kanai for their secretarial assistance. Supported by Grants-in-Aid from the Japanese Ministry of Education, Culture, Sports, Science and Technology (T.F.).

\section{References}

1 Leitch EF, Chakrabarti M, Crozier JEM, McKee RF, Anderson $\mathrm{JH}$, Horgan PG and McMillan DC: Comparison of the prognostic value of selected markers of the systemic inflammatory response in patients with colorectal cancer. Br J Cancer 97: 1266-1270, 2007.

2 Koh CH, Bhoo-Pathy N, Ng KL, Jabir RS, Tan GH, See MH, Jamaris $S$ and Taib NA: Utility of pre-treatment neutrophillymphocyte ratio and platelet-lymphocyte ratio as prognostic factors in breast cancer. Br J Cancer 113: 150-158, 2015.

3 Hanahan D and Weinberg RA: Hallmarks of cancer: the next generation. Cell 144: 646-674, 2011.

4 Fujii T, Yajima R, Tabe Y, Yamaguchi S, Tsutusmi S, Asao T and Kuwano H: Elevated C-reactive protein is associated with the tumor depth of invasion but not with disease recurrence in stage II and III colorectal cancer. Hepatogastroenterology 60: 13432347, 2013.

5 Fujii T, Yajima R, Takada T, Sutoh T, Morita H, Yamaguchi S, Tsutsumi S and Kuwano H: Serum albumin and prealbumin do not predict recurrence in patients with breast cancer. Anticancer Res 34: 3775-3779, 2014.

6 DeNardo DG and Coussens LM: Inflammation and breast cancer. Balancing immune response: crosstalk between adaptive and innate immune cells during breast cancer progression. Breast Cancer Res 9: 212, 2007.

7 Gregory AD and Houghton AM: Tumor-associated neutrophils: new targets for cancer therapy. Cancer Res 71: 2411-2416, 2011.

8 Wei B, Yao M, Xing C, Wang W, Yao J, Hong Y, Liu Y and Fu $\mathrm{P}$ : The neutrophil lymphocyte ratio is associated with breast cancer prognosis: an updated systemic review and meta-analysis. Onco Targets Ther 9: 5567-5575, 2016.

9 Ethier JL, Desautels D, Templeton A, Shah PS and Amir E: Prognostic role of neutrophil-to-lymphocyte ratio in breast cancer: a systematic review and meta-analysis. Breast Cancer Res 19: 2, 2017.

10 Nakano $K$, Hosoda $M$, Yamamoto $M$ and Yamashita $H$ : Prognostic significance of pre-treatment neutron: lymphocyte ratio in Japanese patients with breast cancer. Anticancer Res 34: 3819-3824, 2014.

11 Noh H, Eomm M and Han A: Usefulness of pretreatment neutrophil to lymphocyte ratio in predicting disease-specific survival in breast cancer. J Breast Cancer 16: 55-59, 2013.
12 Azab B, Bhatt VR, Phookan J, Murukutla S, Kohn N, Terjanian $\mathrm{T}$ and Widmann WD: Usefulness of the neutron-to-lymphocyte ratio in predicting short-and long-term mortality in breast cancer patients. Ann Surg Oncol 19: 217-224, 2012.

13 Fletcher JW, Djulbegovic B, Soares H, Siegel BA, Lowe VJ, Lyman GH, Coleman RE, Wahl R, Paschold JC, Avril N, Einhorn LH, Suh WW, Samson D, Delbeke D, Gorman M and Shields AF: Recommendations on the use of F18-FDG PET in oncology. J Nucl Med 49: 480-508, 2008.

14 Fujii T, Yajima R, Yamaguchi S, Tsutsumi S, Asao T and Kuwano $\mathrm{H}$ : Is it possible to predict malignancy in cases with focal thyroid incidentaloma identified by ${ }^{18} \mathrm{~F}$-fluorodeoxyglucose positronemission tomography? Am Surg 78: 141-143, 2012.

15 Flanagan FL, Dehdashti F and Siegel BA: PET in breast cancer: Semin Nucl Med 28: 290-302, 1998.

16 Groves AM, Shastry M, Ben-Haim S, Kayani I, Malhotra A, Davidson T, Kelleher T, Whittaker D, Meagher M, Holloway B, Warren RM, Ell PJ and Keshtgar M: Defining the role of PET-CT in staging early breast cancer. Oncologist 17: 613-619, 2012.

17 Cooper KL, Harnan S, Meng Y, Ward SE, Fitzgerald P, Papaioannou D, Wyld L, Ingram C, Wilkinson ID and Lorenz E: Positron emission tomography (PET) for assessment of axillary lymph node status in early breast cancer: A systematic review and meta-analysis. Eur J Surg Oncol 37: 187-198, 2011.

18 Fujii $\mathrm{T}$, Yajima R, Tatsuki $\mathrm{H}$, Oosone $\mathrm{K}$ and Kuwano $\mathrm{H}$ : Implication of ${ }^{18} \mathrm{~F}$-fluorodeoxyglucose uptake of affected axillary lymph nodes in cases with breast cancer. Anticancer Res 36: 393-397, 2016.

19 Hodgson NC and Gulenchyn KY: Is there a role for positronemission tomography in breast cancer staging? J Clin Oncol 26: 712-720, 2008.

20 Fujii T, Yajima R, Tatsuki $H$, Oosone $\mathrm{K}$ and Kuwano $\mathrm{H}$ : Implication of atypical supraclavicular F18-fluorodeoxyglucose uptake in patients with breast cancer: association between brown adipose tissue and breast cancer. Oncol Lett 14: 7025-7930, 2017.

21 Fujii T, Yajima R, Tsuboi M, Higuchi T, Obayashi S, Tokiniwa H, Nagaoka R, Takada D, Horiguchi $J$ and Kuwano H: Clinicopathological features of cases with primary breast cancer not identified by ${ }^{18}$ F-FDG-PET. Anticancer Res 36: 3019-3022, 2016.

22 Gil-Rendo A, Martinez-Regeira F, Zornoza G, Garcia-Velloso MJ, Beorlegui C and Rodriguez-Spiteri N: Association between ${ }^{18} \mathrm{~F}-$ fluorodeoxyglucose uptake and prognostic parameters in breast cancer. Br J Surg 96: 166-170, 2009.

23 Groheux D, Giacchetti S, Moretti J-L, Porcher R, Espie M, Lehmann-Che J, de Roquancourt A, Hamy AS, Cuvier C, Vercellino L and Hindié E: Correlation of high ${ }^{18}$ F-FDG uptake to clinical, pathological and biological prognostic factors in breast cancer. Eur J Nucl Med Mol Imaging 38: 426-435, 2011.

24 Ueda S, Tsuda H, Asakawa H, Shigekawa T, Fukatsu K, Kondo $\mathrm{N}$, Yamamoto $\mathrm{M}$, Hama $\mathrm{Y}$, Tamura $\mathrm{K}$, Ishida $\mathrm{J}$, Abe $\mathrm{Y}$ and Mochizuki H: Clinicopathological and prognostic relevance of uptake level using ${ }^{18} \mathrm{~F}$-fluorodeoxyglucose positron-emission tomogramphy/computed tomogramphy fusion imaging $\left({ }^{18} \mathrm{~F}-\mathrm{FDG}\right.$ PET/CT) in primary breast cancer. Jpn J Clin Oncol 38: 250258, 2008. 
25 Zhang J, Jia Z, Ragaz J, Zhang YJ, Zhou M, Zhang YP, Li G, Wang BY, Wang ZH and Hu XC: The maximum standardized uptake value of ${ }^{18} \mathrm{~F}-\mathrm{FDG}$ PET scan to determine prognosis of hormone-receptor positive metastatic breast cancer. BMC Cancer 13: 42, 2013.

26 Sanli Y, Kuyumcu S, Ozkan ZG, Isik G, Karanlik H, Guzelbey B, Turkmen C, Ozel S, Yavuz E and Mudun A: Increased FDG uptake in breast cancer is associated with prognostic factors. Ann Nucl Med 26: 345-350, 2012.

27 Allred DC, Harvey JM, Berardo M and Clark GM: Prognostic and predictive factors in breast cancer by immunohistochemical analysis. Mod Pathol 11: 155-168, 1998.

28 Choi JY, Lee KS, Kim HJ, Shim YM, Kwon OJ, Park K, Baek $\mathrm{CH}$, Chung JH, Lee KH and Kim BT: Focal thyroid lesions incidentally identified by integrated ${ }^{18} \mathrm{~F}-\mathrm{FDG}$ PET/CT: Clinical significance and improved characterization. J Nucl Med 47: 609615, 2006.

29 Suppan C, Bjelic-Radisic V, La Garde M, Groselj-Strele A, Eberhard K, Samonigg H, Loibner H, Dandachi N and Balic M: Neutrophil/lymphocyte ratio has no predictive or prognostic value in breast cancer patients undergoing preoperative systemic therapy. BMC Cancer 15: 1027, 2015.

Received June 7, 2018

Revised June 18, 2018

Accepted July 3, 2018 